\title{
Evaluation of Serum Levels of Nesfatin-1 and Insulin resistance in Patients with Acne Vulgaris
}

F.M.Elessawy $^{1}$, A.I.Mostafa ${ }^{1}$, R.A.Khashaba ${ }^{2}$ and A.M.Hamed ${ }^{3}$

${ }^{1}$ Dermatology, Venereology and Andrology Dept., Faculty of Medicine, Benha Univ., Benha, Egypt

${ }^{2}$ Clinical and Chemical pathology Dept., Faculty of Medicine, Benha Univ., Benha, Egypt

${ }^{3}$ Benha Dermatology Clinic Dept., Faculty of Medicine, Benha Univ., Benha, Egypt

E-Mail: Ayatolba3008@gmail.com

\begin{abstract}
Insulin resistance (IR) is identified as an impaired biologic response to insulin stimulation of target tissues, primarily liver, muscle and adipose tissue. Insulin resistance impairs glucose disposal, resulting in a compensatory increase in beta-cell insulin production and hyperinsulinemia. There was a positive correlation between insulin, homeostatic model assessment of insulin resistance (HOMA-IR) values and acne vulgaris. Nesfatin-1 is a novel peptide hormone that suppresses appetite through its actions in the central nervous system. It has anorexigenic effects and it has a major regulatory role in food intake, energy homeostasis, water intake and body temperature. The current study aimed to assess and measure the serum level of nesfatin- 1 and IR in acne vulgaris patients and compare their levels in healthy controls. Fifty patients suffering from acne vulgaris, in addition to thirty apparently healthy individuals of matched age, sex and BMI as a control group. Serum insulin,FBS and nesfatin levels were estimated using commercially available ELISA kits. Our findings suggest that, nesfatin-1 is associated with the susceptibility of development of acne vulgaris and HOMA-IR has a role in the disease pathogenesis and its severity.
\end{abstract}

Keywords: Acne vulgaris, Insulin resistance, Nesfatin-1.

\section{Introduction}

Acne vulgaris (AV) is a cutaneous chronic inflammatory disorder with complex pathogenesis [1]. It is characterized by the development of seborrhea, comedones, nodules, papules, pustules and cyst. It comes in the areas of the skin with plentiful hair growth such as in the upper chest, back, legs and face [2].

Causes of acne include (Use of medications like lithium, steroids and anticonvulsant- exposure to excess sunlight - use of occlusive wear like shoulder pads, headbands and backpacks - endocrine disorders like polycystic ovarian syndrome and even pregnancy) [3].

Acne pathophysiology occurs during puberty under the influence of androgens, sebum secretion is increased as 5-alpha reductase converts testosterone to more potent dihydrotestosterone which binds to specific receptors in the sebaceous glands increasing sebum production. This leads to an increased hyperproliferation of follicular epidermis, so there is retention of sebum. Distended follicles rupture and release pro-inflammatory chemicals into the dermis, stimulating inflammation [4].

Acne may appear in adolescence and it persists through the early thirties. It is more common in males than in females. Urban populations are more affected than rural populations [5].

Acne incidence has been related to changes in insulin and IGF-1 circulating levels rather than to changes in androgen levels. During puberty and adolescence, there is a reduction in insulin sensitivity, combined with an increase in IGF-1 and insulin serum levels and a decrease in SHBG and IGFBP-1 serum levels. In particular, insulin and IGF-1 levels peak during late puberty and gradually decline until the third decade. Acne begins about the same time as the preadolescent increase in plasma insulin, IGF-1 and generally resolves by the end of puberty despite circulating androgens remain unchanged [6].

Patients with acne often exhibit increased levels of serum glucose and insulin as well as insulin resistance and its association has been long recognized [7]. There was a positive correlation between insulin, HOMA-IR values and AV [8]. As regard to assessment of insulin level in diseases other than AV. Insulin resistance was high in women with PCOS. It is one of the key diagnostic features of PCOS which manifests clinically as acne [9].

Nesfatin-1 is a recently discovered anorexigen encoded in the precursor peptide, nucleobindin-2 (NUCB2) in the hypothalamus of mammals and participates in the regulation of hunger and fat storage [10]. NUCB2 is expressed both in the CNS as well as in peripheral tissues and it has been shown that nesfatin-1 can also cross the blood-brain barrier [11].

Nesfatin-1 plays a role in energy homeostasis and metabolism. It was reported to be able to pass through the blood brain barrier and to produce anorectic responses after its administration to rodents at pharmacological doses [11]. Central nesfatin-1 is involved in controlling the stress axis, and nesfatin-1 expression is subject to the negative feedback regulation of the main secretory products of the adrenal gland [12].

Therefore, additional studies on the expression and subcellular localization of nesfatin and insulin resistance in relation to function will confirm their key roles in the skin and will open the field to new therapeutic strategies for many cutaneous conditions such as acne scaring. 


\section{Materials and methods}

\subsection{The Study population}

This is a case-control study . The current study had been conducted in June and July 2018 after the approval by Research Committee at Faculty of Medicine, Benha University. The study included 80 subjects that were randomly selected from those attending the Dermatology Outpatient's Clinic at Benha University Hospital, Faculty of Medicine, Benha University, Egypt. Prior to initiation of the study, every subject was informed about the aim of the study and an informed consent was obtained from each individual before sample collection. They were classified into two groups; the first group included 50 patients suffering from acne with no history of receiving any treatment that affect blood glucose level, any eating disorders, any acute or chronic infection, any thyroid disorders, any chronic renal or liver diseases and the second group consisted of 30 healthy subjects to serve as a control group.

\subsection{ELISA assays of nesfain-1}

A double-antibody sandwich Enzyme Linked Immune Sorbent Assay (ELISA) was used to detect serum level of nesfatin using a commercial Human Nesfatin ELISA Kit for research use only (Cat \#: 201-12-4341, Sun Red Bio, China).

\subsection{Statistical analysis}

Table (1) Comparison between serum Nesfatin-1 levels between both studied groups.
The collected data was revised, coded, tabulated and introduced to a PC using Statistical package for Social Science (IBM Corp. Released 2011. IBM SPSS Statistics for Windows, Version 20.0. Armonk, NY: IBM Corp.). Data were presented and suitable analysis was done according to the type of data obtained for each parameter. Student T Test was used to assess the statistical significance of the difference between two study group means. For the comparison of the three groups' means, one way analysis of variance (ANOVA) was used. Chi-Square test was used to examine the relationship between two qualitative variables Fisher's exact test: was used to examine the relationship between two qualitative variables when the expected count is less than 5 in more than $20 \%$ of cells. Correlation analysis: To assess the strength of association between two quantitative variables. The correlation coefficient defines the strength and direction of the linear relationship between two variables. The correlation coefficient defines the strength and direction of the linear relationship between two variables. $p$ is significant if $<0.05$ at confidence interval $95 \%$.

\section{Results}

\subsection{The Study Population}

serum insulin level between both groups ( $\mathrm{P}$ value $0.137,0.201,<0.001)$ respectively as shown in Table (1).

\begin{tabular}{|c|c|c|c|c|}
\hline Variable & & $\begin{array}{c}\text { Patients } \\
\mathrm{N}=\mathbf{5 0}\end{array}$ & $\begin{array}{c}\text { Control } \\
\mathrm{N}=30\end{array}$ & $\mathbf{P}$ \\
\hline Nesfatin-1 (ng/mL) & $\begin{array}{c}\text { Mean } \pm \text { SD } \\
\text { Median (Min-Max) }\end{array}$ & $\begin{array}{c}3 \pm 1.7 \\
1-14\end{array}$ & $\begin{array}{c}18 \pm 5.4 \\
4-77\end{array}$ & $0.001^{\mathrm{T}}$ \\
\hline
\end{tabular}

Mean nesfatin-1level in control group was 27.6 $\mathrm{ng} / \mathrm{mL}$, while it was $4.8 \mathrm{ng} / \mathrm{mL}$ in patients. Acne vulgaris group showed significantly lower serum nesfatin-1 level when compared to control group ( $\mathrm{p}$ value 0.001 ) as shown in Table (1).

\section{Discussion}

Acne Vulgaris is a chronic inflammatory disease of the pilosebaceous unit, characterized by formation of comedones, erythematous papules, and pustules, less frequently by nodules, deep pustules, or pseudocysts and, in some cases, it is accompanied by scarring [13].

This case-controlled study was designed to measure the serum level of nesfatin-1 and IR in AV patients and compare their levels in healthy controls.

Our study showed that, face was affected in all cases, back in $38 \%$ and chest in $12 \%$. This was in agreement with another study which found that a high proportion of sebaceous glands found on the face $99 \%$, back $60 \%$ and chest $15 \%$ [14]. Also, [15] suggested that face alone was involved in $65.4 \%$ of the cases. This was followed by the involvement of the back $28.2 \%$, chest $20.1 \%$, neck $9.4 \%$ and arms $10 \%$.

Acne Vulgaris group showed significantly higher HOMA-IR, when compared to control group. In agreement with the present study, a study conducted by [16] who described that, there was a highly significant difference of HOMA-IR values between the patient and control groups. [17] found that HOMA-IR values were significantly higher in patients with AV when compared with the control group. While these results disagreed with other studies done by [18] who said that, the difference among the mean values of HOMA-IR were not statistically significant.

There were no significant differences in FBG and serum insulin level between both groups. These data came in agreement with previous studies done by [18] who found that, there were statistically no significant differences between cases and control regarding FBG and insulin. Also, [19] has not shown any significant difference in FBG levels between patients with AV and controls. On contrary to these 
results, there were previous studies done by [17] who described that, insulin and FBG values were significantly higher in patients with acne when compared with the control group.

Acne Vulgaris group showed significantly lower serum nesfatin-1 level when compared to control group. To the best of our knowledge, no published studies were found to compare the results between AV patients concerning serum nesfatin-1levels. Results of the present study showed statistically insignificant difference of serum nesfatin level in AV patients regarding age and BMI.

\section{Conclusions}

From the results of present study, it is concluded that IR is more common among AV patients especially in patients with severe AV lesions and that could be explained by lower serum nesfatin-1 level in those patients. Since acne is a problem in adolescents, the early recognition of IR might help in better management of acne patients.

\section{Recommendations}

The results of our study should be interpreted in light of its limitations, as the present study included a relatively small sample size. Further studies are needed to investigate the precise mechanisms by which nesfatin-1 contribute to the pathogenesis of AV. Nesfatin is needed to be measured in tissue also and needed to be measured before and after treatment.

\section{Conflict Of Interest}

The authors declare that they have no conflict of interest.

\section{References}

[1] T.Cong, D.Hao, X.Wen, From pathogenesis of acne vulgaris to anti-acne agents. Arch Dermatol Res, Vol.311, PP.337-349, 2019.

[2] B.Ravisankar, H.Liu, R.Shinde, The amino acid sensor GCN2 inhibits inflammatory responses to apoptotic cells promoting tolerance and suppressing systemic autoimmunity, Vol 112, PP.10774-10779, 2015.

[3] H.Sutaria and J.Schlessinger, Acne Vulgaris. Nut, Vol.10, PP.29-39, 2018.

[4] O.Alexeyev, I.Dekio, A.Layton, Why we continue to use the name Propionibacterium acnes. Br J Dermatol, Vol.179(5), PP.12231227, 2018.

[5] S.Ozçelik, İ.Kulaç, M.Yazıcı, Distribution of childhood skin diseases according to age and gender, a single institution experience. Turk Ped Ars, Vol.53, PP.105-112, 2018.

[6] R.Smith, N.Mann, A.Braue, The effect of a high-protein, low glycemic-load diet versus a conventional, high glycemic-load diet on biochemical parameters associated with acne vulgaris: a randomized, investigator-masked, controlled trial. J Am Acad Dermatol, Vol.57, PP. 247-256, 2007.

[7] B.Melnik, S.John and G. Plewig,Acne: risk indicator for increased body mass index and insulin resistance. Acta Derm Venereol, Vol.93, PP.644-649, 2013.

[8] Demir B, Ucak H, Cicek D, Changes in serum desnutrin levels in patients with acne vulgaris. Eur J Dermatol, Vol.24, PP.589-593, 2014.

[9] A.Vryonidou, S.Paschou, G.Muscogiuri, Metabolic Syndrome through the female,s life cycle. Eur J Endocrinol, Vol.1, PP.133-157, 2015.

[10] Gonzalez R, Kerbel B, Chun A, Molecular, Cellular and Physiological Evidences for the Anorexigenic Actions of Nesfatin-1 in Goldfish. PLoS ONE, Vol.5, PP.:1-14, 2010.

[11] W.Pan, H.Hsuchou and A.Kastin , Nesfatin-1 crosses the bloodbrain barrier without saturation.Peptides, Vol.28, PP.2223-2228, 2015.

[12]M.Yang, Z.Zhang, C.Wang, Nesfatin-1 action in the brain increases insulin sensitivity through Akt/AMPK/TORC2 pathway in dietinduced insulin resistance.Diab, Vol.61, PP.1959-1968, 2012.

[13] N.Simpson and W. Cunliffe (): Disorders of the sebaceous glands. Rook's Text Book of Dermatology, Vol.7(43), PP.1-7, 2004.

[14] S.Rahaman, D.De and S. Handa, Association of insulin-like growth factor (IGF)-1 gene polymorphisms with level of IGF-1 and acne severity. J Am Acad Dermatol, Vol.75, PP.768773, 2016.

[15]B.Adityan and D. Thappa (): Profile of acne vulgaris-A hospital-based study from South India. Indian J Dermatol Venereol Leprol, Vol.75, PP.272-278, 2009.

[16] N.Emiroğlu, F.Cengiz and F. Kemeriz, Insulin resistance in severe acne vulgaris. Postepy Dermatol Alergol, Vol.32, PP.281-285, 2015.

[17] D.Kartal, H.Diz, R.Ertas, Association between isolated female acne and insulin resistance: a prospective study. Italy J Dermatol Venereol, Vol.151, PP.353-357, 2016.

[18] M.Abdelmawla, A.Esawy, E.Khater, Insulin resistance in androgenetic alopecia and acne vulgaris. Egy J Dermatol Venerol, Vol.39, PP.83-88, 2019.

[19]I.Balta, O.Ekiz, P.Ozuguz, Insulin resistance in patients with post-adolescent acne. Int $\mathbf{J}$ Dermatol, Vol.54, PP.662-666, 2015. 